\title{
Patient Recruitment by Neurological Registries
}

\author{
Mark Hamilton ${ }^{1}$, Angela Genge ${ }^{2}$, Megan Johnston ${ }^{1}$, Darren Lam ${ }^{1}$, \\ Theo Mobach ${ }^{1}$, James Marriott ${ }^{3}$, Thomas Steeves ${ }^{4}$, Elizabeth Donner ${ }^{4,5}$, \\ Julie Wysocki', Karen Barlow ${ }^{1}$, Michael Shevell ${ }^{2}$, Ruth Ann Marrie ${ }^{3}$, \\ Steve Casha ${ }^{1}$, Gail MacKean ${ }^{1}$, Lisa Casselman ${ }^{1}$, Lawrence Korngut ${ }^{1}$, \\ Tamara Pringsheim ${ }^{1}$, Nathalie Jette ${ }^{1,7,8}$
}

Can J Neurol Sci. 2013; 40: Suppl. 2 - S23-S26

This section summarizes the considerations surrounding patient recruitment that Canadian neurological registries should address during planning and design. In preparation of this guideline, we examined relevant Canadian and international literature; Canadian policy and legislation. We also consulted with Canadian privacy officers and specialists in research ethics.

\section{BACKGROUND}

Clinical registries capture patient information contingent upon successful recruitment and retention of patients who will consent to participation. To accomplish this requires the elements that affect patient recruitment. For example, failure to adequately engage physicians or other healthcare professionals can have as much impact on recruitment success as failure to adequately identify the patients relevant to the purposes of the registry. A strategy for recruitment that is not properly targeted to relevant patients will fail to provide desired information.

\section{RELEVANT LITERATURE}

A literature review identified 96 abstracts describing registry recruitment. Full text reviews were performed on 37 articles and identified 23 articles for summarization.

\section{General Overview}

Recruitment in a comprehensive manner can result in population-based registries that are highly generalizable and can be used for the identification of eligible participants for future research studies.53-56 Those involved in the creation of population-based registries should be aware that recruitment and participant biases occur when individuals that consent or refuse to participate are inherently different from the population as a whole..$^{53,55,57,58}$ Such biases in registries may result in unrepresentative or non-generalizable data thus it is important to ensure that recruitment strategies are effective and that the resulting sample is representative of the target population.
Although recruitment of clinic-based populations may prove to be more successful with respect to retention, using a populationbased sampling frame offers the methodological advantage of recruiting a representative sample. ${ }^{59}$ Recruitment and enrollment into a registry may be mandatory for certain conditions such as Creutzfeld-Jacob Disease, ${ }^{17,53-56,60}$ yet many other diseases require patient consent to enroll into the registry. Barriers to development of population-based registries exist but specific strategies have been shown to be effective in both recruitment and retention.

The Agency for Healthcare Research and Quality (ARHQ) Manual "Registries for Evaluating Patient Outcomes A Users Guide" 5 is a valuable resource that critiques strategies for retention and recruitment. The validity of registry data may be profoundly compromised if common problems associated with clinical studies are not addressed (e.g. difficulties with patient enrollment, losses to follow-up, and certain sites contributing most patients). Generally, the burden of participation should be minimized, while the relative rewards, particularly nonmonetary rewards, should be maximized. One must be aware of the use of confusing terminology (i.e. it is critical that the language and terminology are clear and concise) as a potential further source of recruitment bias.

\section{Ethnic Diversity \& Other Barriers toward Recruitment}

Mitigation of factors that may result in selection bias requires consideration of ethnic diversity. Fear of foreign medical institutions and skepticism related to research might prevent certain groups from enrolling in registries. ${ }^{21,61}$ Bachman et $\mathrm{al}^{61}$ postulated a number of reasons that may contribute to the difficulties in recruiting certain ethnic groups for research purposes: Information about research not reaching the community, the perception that research is biased to benefit the white population, insufficient community involvement by the research team to allow trust to develop, concerns that the research is not relevant to their community, the lack of use of

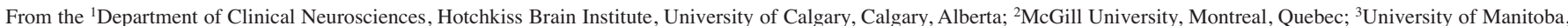
Winnipeg, Manitoba; ${ }^{4}$ University of Toronto, Toronto, Ontario; ${ }^{5}$ Hospital for Sick Children, Toronto, Ontario; ${ }^{6}$ Parkinson Society of Canada, Toronto, Ontario; ${ }^{7}$ Institute for Public

Health, University of Calgary, Calgary, Alberta.

Final REvisions SubmitTEd JanUARY 28, 2013

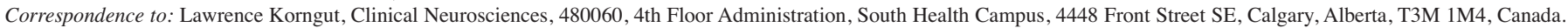

Email: Lawrence.korngut@albertahealthservices.ca. 
existing networks and failure to advertise in appropriate locations, the lack emphasis to the community regarding the importance of research, inadequate compensation for participation, the possibility that the research does not address a personal or family medical problem, inadequate representation of minorities on the research team, and limited time for healthcare-related activities for potential participants and family members. Other documented reasons for recruitment refusal include not wanting to travel to participate, feeling unable to commit, and lack of interest. ${ }^{53}$ Physicians are often involved in the recruitment process $53,54,60-62$ and some have noted that involving their patients in a registry involves time commitment and intrusion of the study on the physician-patient relationship ${ }^{53}$ - a potential barrier to recruitment. Other limitations include geographical remoteness and related transportation costs. ${ }^{21}$

\section{Vulnerable Populations}

Within neurological conditions it is not uncommon to seek enrollment of patients who may be members of a vulnerable population. Such vulnerable populations may include minors; individuals with mental or cognitive difficulties; and individuals with low socioeconomic status. With respect to issues of capacity (minors and individuals with cognitive impairment) more information can be found in the Ethical and Legal considerations section of this document. Addressing recruitment strategies toward individuals with low socioeconomic status may be more challenging. Assessment of the relative or expected socioeconomic status in the registry's target population is a critical step toward ensuring recruitment strategies can be inclusive of participants with low socioeconomic status. Additionally, it may be helpful to obtain statistics on the socioeconomic profile of patients attending clinics where recruitment will occur to ensure a representative sample can be obtained. Finally, utilizing multiple recruitment modalities may help to reach vulnerable populations.

\section{Sources and Methods of Patient Recruitment}

A critical aspect of any successful registry involves the correct identification of the relevant patient population. This process begins by defining the diagnostic characteristics and how the population will be accurately identified. The patient population may, for example, be predominately diagnosis-based (e.g. Multiple Sclerosis) vs. treatment-based (e.g. anti-epileptic medications) or hospital-based vs. community physician-based. Once the location and characteristics of the population are established, recruitment methodologies can be addressed. As examples, patients may potentially be approached after reviewing diagnostic codes associated with medical records or other administrative data sources, or in association with visits to family practice or specialty care clinics.

Recruitment of individuals meeting eligibility criteria has been accomplished through a myriad of strategies - both active and passive. Active strategies include recruitment through medical staff and clinical sites ${ }^{11,56,58-61,63}$, searching through health records, ${ }^{16,55,57,60}$ seeking participants in specific community locations (e.g. senior housing venues, senior co-op housing, city senior services) ${ }^{64}$ or reaching out to the community by providing educational learning series ${ }^{63,64}$. Passive strategies include attracting patients through the Internet and websites, ${ }^{17,23,57,62}$ media and awareness campaigns, ${ }^{17,55,57,63,65,66}$ and information brochures, flyers, or both ${ }^{17,23,57,63-65}$ In several prior studies, a toll-free information contact number on brochures/flyers and media and awareness campaigns were provided to interested individuals. ${ }^{57,63}$

\section{Additional Points Regarding Physician and Patient Recruitment and Retention}

The overall success of a patient registry is largely dependent on the successful recruitment of patients. However, this requires the engagement of patients and physicians or other healthcare professionals. The importance of this element should not be underestimated. Strategies to involve physicians and other healthcare professionals include providing a clear representation of the registry structure, methods and standard operating procedures (SOPs) so as to avoid any process confusion; and the development of strategies to keep the process of patient recruitment and data collection as simple as possible. It is equally important to keep health professionals engaged in the process by providing regular updates. The success of a registry is enhanced by providing adequate resources to support the recruitment of patients, and the collection, verification and entry of data. While it is often challenging to obtain funds for registries, this should be a goal.

A lack of physician experience with research may also impact successful patient recruitment into registries. To provide for long-term registry viability it may be wise to try to team a senior member (physician) to act as a mentor with a junior member. The establishment of a clear business plan with a detailing of any financial resources available and financial obligations for physician participants is also critical to avoid surprises that may discourage physician recruitment or retention in a registry.

During the process of establishing a registry, it could be useful to hold multiple focus groups inviting both healthcare professionals and patients to participate. This would allow for assistance in identifying key registry issues before methodology and SOPs are established. During this process, stakeholder organizations should be engaged to obtain their input regarding how to improve patient recruitment and retention strategies.

\section{Ethical Recruitment}

It is vital to ensure that recruitment is accomplished with clear regard to all ethical considerations. Strategies for recruitment and retention should be vetted for practical and ethical concerns, developed into SOPs and implemented with monitoring to ensure compliance. These strategies should deal with (but not be limited to) such issues as where and how patients can be approached (e.g. clinic vs. letter), who can approach patients (e.g. clinician vs. research team member), whether it is suitable to pay transportation costs for patients who participate in a registry, and how to manage the recruitment of vulnerable populations such as the cognitively impaired.

Also, as part of the development of SOPs, clear guidelines must be established which define who has access to data entry, data review and data analysis for a registry. This information (i.e. the clearly developed and vetted SOPs) can be communicated with patients so that they have a clear understanding of their responsibilities and trust regarding the safety and security of their personal information. 
More detail on ethical recruitment considerations can be found in the Ethical and Legal considerations section of this document.

\section{Maximizing Enrollment}

Several proposed strategies may help achieve a high registry participation rate. First, using a variety ${ }^{17,65}$ of recruitment strategies can improve the participation rate. Wei et al's InternetBased Clinical Trials Database for colorectal cancer showed that $88 \%$ of patients registered through the Internet as opposed to $12 \%$ through the call center, supporting the efficacy and usefulness of the Internet for recruitment. ${ }^{17}$ Gupta et al further supports the idea of Internet-based patient recruitment because it represents an opportunity for efficient recruitment of patients for rare lung disease studies. ${ }^{21}$ However, internet registration may not be effective at reaching all age groups or demographics, therefore there is utility to using multiple approaches to ensure a representative sample can be obtained. The adoption of technology may aid recruitment process for patients - this can involve the use of online questionnaires ${ }^{18,27}$ or using touchscreen computers. ${ }^{27}$ More detail can be found in the Online Registries section of this document. Involving treating physicians that have established a good physician-patient relationship can also be effective for recruiting participants. ${ }^{16,20,54}$ Providing clear information in advance so patients have time to raise questions about the study, explaining the benefits of participation, clarifying how the costs to the participants will be covered, ensuring that the patient is aware of the confidential nature of the study, ensuring that the patient understands that they have the ability to withdraw at any time, and supplying local media with stories that will raise the profile of the study are all strategies that can help achieve a high enrolment rate. ${ }^{20}$ To improve recruitment, Gupta et $\mathrm{al}^{21}$ suggested providing benefits with registry membership (such as access to disease forums and information resources), and using clinical research networks and organizations. Newberry et $\mathrm{al}^{58}$ concluded that several specific recruiter and interviewer training techniques were associated with higher recruitment and retention: increased communication, becoming familiar with the community and recruitment sites, being flexible with recruitment approaches, being aware of cultural differences in participation, and the timing of the approach in relation to the initial diagnosis (higher chance of refusal if approached too soon after diagnosis) may impact willingness to participate in neurology research. Sending a post-card and a phone call following initial contact resulted in the best patient response rates in the Ontario Familial Breast Cancer Registry. ${ }^{60}$

\section{Reducing Attrition}

Following successful recruitment, patients may be lost to follow-up over time. It is necessary to implement strategies that limit attrition. Loss of follow-up tends to be highest in those registries relying on voluntary reporting through healthcare providers where incentives for complete reporting are not provided ${ }^{67}$ Golding et $\mathrm{al}^{20}$ recommend obtaining contact information of one or two individuals who would be likely to know the new location of the study participant/family in the situation that they relocate or are lost to clinical follow-up. Reminders such as fridge magnets ${ }^{66}$ or phone calls ${ }^{58}$ can also help limit attrition.
A critical factor in retention is delivery on promises made during recruitment (i.e. that the burden of patient participation is low). Provider participation retention tools include: Web sites, newsletters, telephone helplines, instruction manuals, training meetings, site audit/retraining visits, satisfaction/opinion surveys, regular data reports to stakeholders, presentations at conferences, regular reports to registry participants on registry growth and publications, and the ability of participating physicians to publish based on registry data. Retaining patients require the development of a retention plan. For patients who transfer to non-enrolled practices, enlisting site staff to reach out to patients beyond their standard interactions, following patients directly through a central patient management center, and linking to other data sources to obtain key long-term outcomes data on patients who are lost to follow-up is essential. ${ }^{5}$

\section{Special Considerations in Canada}

There is literature regarding recruitment strategies for hospitals that does not have any significant practical context in Canada.

Registries may be centered in one jurisdiction or span multiple jurisdictions which requires that careful attention be paid to the legislative requirements and privacy considerations for each jurisdiction (e.g. province) that is involved. This may become more complex if data-linkage with health system administrative data is planned.

Potential Canadian-specific sources for patient recruitment include provincial home-care networks (which may capture people not identified in clinics), public health clinics, and in Quebec, the CLSCs (centre local de services communautaires or local community service centres) where patients may not directly interact with a physician. Non-physician sources of recruitment such as rehabilitation centres or allied healthcare practitioners (e.g. occupational therapists, physical therapists; dietitians etc) should also be considered.

Specific Canadian cultural considerations must be addressed while developing a registry to ensure that all population characteristics are represented. One specific aspect relates to the benefits of bilingual recruitment, which allows researchers to reach out to a more diverse population. This may involve increased cost and challenges with regard to providing seamless access to interpreters and professionally translated materials.

When determining the registry population to be targeted, it also is important to consider Aboriginal groups which otherwise might be underrepresented or missed with conventional recruitment strategies. This will require a clear understanding of Aboriginal policies and legalities so that appropriate representative bodies are involved. More information on considerations for registries working with Aboriginal populations can be found in the Ethical and Legal considerations section of this document. 


\section{RECOMMENDATIONS}

$\checkmark$ Ensure that physicians and other healthcare professionals are involved as needed, engaged and regularly updated. Consider engaging participants and healthcare professionals during the design phase to assess needs and gather resources.

$\checkmark$ Recruit patients from various sources to ensure that the study population is representative of the total disease population of interest. Address challenges associated with physician/healthcare professional participation and utilize strategies to engage nonacademic physicians/healthcare professionals when appropriate. $\checkmark$ Establish a registry website that can be used as a resource by patients. The registry website may also be used as a recruitment tool.

$\checkmark$ Consider utilization of a patient consent to be contacted about research within routine clinical practice. This can increase the ability to recruit registry patients through phone or letter contact. $\checkmark$ Engage advocacy groups and other stakeholders to encourage participation or reach potential participants that are otherwise inaccessible. $\checkmark$ Minimize participant and clinician burdens of participation, especially time.

$\checkmark$ Ensure every registry site / jurisdiction has its own representative and champion as well as adequate resources (e.g. nursing staff support; financial etc.).

$\checkmark$ Develop and test SOPs outlining recruitment strategies and procedures. Ensure that these are reviewed by a research ethics board.

$\checkmark$ Ensure that registry participants and healthcare professionals feel as though they belong to a group.

$\checkmark$ Where bilingual or multi-lingual recruitment is desired, ensure that recruitment documents and procedures address the appropriate language needs.

$\checkmark$ Ensure that recruitment strategies address the needs of special populations (e.g. pediatric assent strategies; considerations for Aboriginal populations etc.). 